\title{
A mechanism for shape coexistence
}

\author{
Andriana Martinou ${ }^{1 *}$ \\ ${ }^{1}$ Institute of Nuclear and Particle Physics, National Centre of Scientific Research "Demokritos", GR-15310 Aghia Paraskevi, Attiki, \\ Greece
}

\begin{abstract}
The phenomenon of shape coexistence in a nucleus is about the occurence of two different nuclear states with drastically different shapes, lying close in energy. It is commonly seen in the data, that such coexisting states manifest in specific nuclei, which lie within certain islands on the nuclear chart, the islands of shape coexistence. A recently introduced mechanism predicts that these islands derive from the coexistence of two different types of magic numbers: the harmonic oscillator and the spin-orbit like. The algebraic realization of the nuclear Shell Model, the Elliott $S U(3)$ symmetry, along with its extension, the proxy-SU(3) symmetry, are used for the parameter-free theoretical predictions of the islands of shape coexistence.
\end{abstract}

\section{Introduction}

The studies about shape coexistence began with the excited $0_{2}^{+}$state of ${ }^{16} \mathrm{O}$ [1]. It was then suggested that this excited state of ${ }^{16} \mathrm{O}$ can be explained by a particle-hole (p-h) excitation across the harmonic oscillator shell among the magic numbers 2-8. This suggestion triggered the idea of particle-hole excitations, which became later on the stateof-the-art mechanism for shape coexistence [2].

Several years later, another phenomenon, that of the inversion of states, became known in the region of the $\mathrm{Mg}$ isotopes [3-7]. Inversion of states occurs, when the predicted spin and parity of the ground state contradicts with the data. Specifically the predictions are produced with the use of the Shell Model [8,9] and with the assumption that the particles occupy the valence harmonic oscillator shell, which lies among the magic numbers 8-20 in the Mg isotopes.

The intense study in this region led to the conclusion that the inversion of states begins and ends at a certain neutron number $N$ across an isotopic chain [3]. Thus discussion about the "islands of inversion" began [10, 11]. Both theorists and experimentalists drew the conclusion that the inverted states are due to a partial particle configuration in the valence harmonic oscillator shell and in the slightly excited spin-orbit like shell $[12,13]$. The spin-orbit like shells [14] lie among the nucleon magic numbers 6,14 , $28,50,82,126, \ldots$, which are created by a significant spinorbit force [9]. So the idea that particles are excited from the the harmonic oscillator shell to the neighboring spinorbit like shell has been established in the light nuclei.

The same idea has been used in Ref. [15] to predict the islands of shape coexistence in the medium mass and heavy nuclei. Again it is supposed that the islands of shape coexistence can be predicted, if one assumes that the particles occupy the valence harmonic oscillator shell along

*e-mail: martinou@inp.demokritos.gr with the valence spin-orbit like shell. But since in medium mass and heavy nuclei, the particles usually already are in the spin-orbit like shells among magic numbers 28,50 , 82,126 , we cannot speak about particle excitations from the valence harmonic oscillator shell to the valence spinorbit like shell. Instead, the situation is better described by the phrase "shell merging" $[12,13]$. Shell merging occurs when the single-particle energy gaps dissolve due to the onset of deformation and thus the valence harmonic oscillator shell merges with the valence spin-orbit like shell. So the key to enter into an island of shape coexistence is the deformation.

Generally the sudden onset of deformation in specific nuclei is attributed to the proton-neutron interaction, when the last protons and neutrons occupy specific Shell Model orbitals, namely the spin-orbit partners or the FedermanPittel pairs introduced in Ref. [16-18]. Actually the particle-hole excitation mechanism, which is the state-ofthe-art mechanism for shape coexistence, is based on the Federman-Pittel pairs (see Ref. [19]). The main idea is that the protons lie near a magic or semi-magic number, above which large single-particle energy gaps extend and that the protons are being excited, so as to create spinorbit partners with the neutrons. The states created by the spin-orbit partners are highly deformed and thus lower in energy. Obviously the magic or semi-magic numbers play a crucial role in the particle-hole excitations mechanism. Thus, in regions where the magic or semi-magic numbers are not justified, the particle-hole excitation mechanism cannot be used as an explanation for the occurrence of shape coexistence, or inversely, where shape coexistence appears, sub-shell gaps have to be justified. For further discussion see for instance subsection IIIA3 of Ref. [2].

In Ref. [15] an alternative mechanism, namely the dual-shell mechanism, has been introduced in order to explain the phenomenon of shape coexistence. This mechanism has similarities with the particle-hole excitation 
mechanism in light nuclei (see section 4 of Ref. [15]) and aligns with the concept of shell merging in medium mass and heavy nuclei (see section 5 of Ref. [15]). The mechanism is realized within the Elliott [20-23] and the proxy-SU(3) [24-28] symmetry. Deformed nuclei are adequately described by the Shell Model $S U(3)$ symmetry, or nowadays called the Elliott $S U(3)$ symmetry [20-23], which is appropriate for the harmonic oscillator shells, along with its extension, the proxy- $S U(3)$ symmetry [2428], which suits to the spin-orbit like shells. These two algebraic realizations of the nuclear Shell Model use the Shell Model single-particle basis, but in the cartesian coordinate system, in order to give parameter-free predictions about the nuclear deformation.

\section{The dual-shell mechanism}

The Elliott $S U(3)$ three-dimensional (3D) isotropic harmonic oscillator (HO) Hamiltonian is the sum [29]:

$$
H_{0}=\sum_{i=1}^{A} h_{0, i},
$$

where $A$ is the mass number and $h_{0 i}$ is the Hamiltonian of a single particle $i$ moving into a three dimensional isotropic harmonic oscillator potential. The overall algebraic quadrupole-quadrupole $(Q Q)$ interaction in the Elliott $S U(3)$ model is calculated by the formula [30, 31]:

$$
Q Q=4 C_{2}-3 L(L+1),
$$

where $L$ is the orbital angular momentum $[20,29]$ and $\hat{C}_{2}$ is the second order Casimir operator of the $S U(3)$ algebra obtaining eigenvalues [23, 32]:

$$
C_{2}=\lambda^{2}+\mu^{2}+\lambda \mu+3(\lambda+\mu),
$$

with $(\lambda, \mu)$ being the quantum numbers of the Elliott $S U(3)$ symmetry. The $C_{2}$ operator is proportional to the square of the nuclear quadrupole deformation parameter $\beta$ of the Bohr and Mottelson Collective Model [33, 34]:

$$
\beta^{2} \sim\left(C_{2}+3\right)
$$

The simplest $S U(3)$ Hamiltonian is [35]:

$$
H=H_{0}-\frac{\chi}{2} Q Q .
$$

The parameter $\chi$ is the strength of the quadrupolequadrupole interaction and is given by a global expression in Ref. [35]:

$$
\chi=\frac{\hbar \omega}{4 N_{0}} .
$$

Alternatively, instead of $\chi$, the parameter $\kappa$ can be used [35]:

$$
\kappa \approx 2 \chi
$$

This doubling of the quadrupole strength is in the same spirit with the scaling factor, used in Ref. [25], for the $\beta^{2}$ values. Generally in the $S U(3)$ nuclear models the deformation parameter $\beta^{2}$ and the $B(E 2) s$ obtain smaller values than those in the data. So, a scaling is to be used and this is justified by the fact that the $S U(3)$ models use the algebraic quadrupole operator, which is restricted within one nuclear shell, while the data are attributed to the collective quadrupole operator, which applies to to many nuclear shells with the same parity. Nevertheless, once the choice of $\chi$ or $\kappa$ is done, the quadrupole strength will be given by a global expression and it is not treated as a free parameter.

Using the Hamiltonian of Eq. (5) the energy difference among the two bandheads of shape coexistence is the eigenvalue of the difference:

$$
\begin{array}{r}
H_{H O}-H_{S O}=\left(H_{0, H O}-H_{0, S O}\right) \\
+\frac{\chi}{2}\left(Q Q_{S O}-Q Q_{H O}\right),
\end{array}
$$

where the subscripts $S O$ and $H O$ stand for the spin-orbit like and the harmonic oscillator valence shells respectively. The difference

$$
\Delta\left(H_{0}\right)=H_{0, H O}-H_{0, S O} \leq 0
$$

obtains negative values [15], while

$$
\Delta\left(\frac{\chi}{2} Q Q\right)=\left(\frac{\chi}{2} Q Q\right)_{S O}-\left(\frac{\chi}{2} Q Q\right)_{H O} \geq 0
$$

is the difference in the quadrupole terms and obtains positive values within the regions of shape coexistence. Thus the two differences, due to their opposite signs, compete with each other in the Hamiltonian (8) and the energies of the two bandheads of shape coexistence lie close in energy. The islands of shape coexistence appear when the deformation prevails [15]:

$$
\Delta\left(\frac{\chi}{2} Q Q\right) \geq-\Delta\left(H_{0}\right) \geq 0
$$

and till the harmonic oscillator shell closure. The above condition applies for the proton or neutron configurations separately. Thus it implies that the proton (neutron) configuration of the spin-orbit like shell lies lower in energy, than the one deriving from the harmonic oscillator shell. But the situation for the coupled proton-neutron configuration is uncertain and it might result that the configuration of the spin-orbit shell lies higher in energy. Thus, the condition $\Delta\left(\frac{\chi}{2} Q Q\right) \geq 0$ leads to:

$$
C_{2, S O}-C_{2, H O} \geq 0
$$

for the proton or neutron configuration separately and gives the widest possible regions of shape coexistence predicted by the dual-shell mechanism.

The values of the $C_{2}$ are determined in Eq. (3) by the $S U(3)$ irrep. In the literature, there exist two kinds of Elliott $S U(3)$ irreps as candidates for the description of the low lying in energy nuclear states: the highest weight irrep $(\lambda, \mu)_{h w}$ and the most deformed irrep $(\lambda, \mu)_{d e f}$. Up to the middle of each valence shell the two irreps coincide $(\lambda, \mu)_{h w}=(\lambda, \mu)_{d e f}$ and consequently the $C_{2}$ operators obtained by them with the use of Eq. (3) are the same, $C_{2, h w}=C_{2, d e f}$. But after the middle of the shell and before the shell closure the two irreps become different $(\lambda, \mu)_{h w} \neq(\lambda, \mu)_{d e f}$ with $C_{2, h w} \leq C_{2, \text { def }}$. In Ref. [25] 
the $(\lambda, \mu)_{d e f}$ is labeled by $C$, which means that it is the irrep with the maximum value of the $C_{2}$ for a given number of protons or neutrons in a given valence shell. In Refs. $[25,28,36]$ it is stressed out that the highest weight irrep has to be used, because this irrep gives the right prolateoblate transition. Consequently the widest possible islands of shape coexistence determined by the condition (12) are predicted with the use of the highest weight irreps for both spin-orbit like shells and harmonic oscillator shells. The relevant irreps are tabulated in Ref. [15].

\section{Results}

The condition (12) is satisfied among the proton or neutron numbers:

$Z$ or $N: 7-8,17-20,34-40,59-70,96-112,145-168$.

These results have been obtained using the highest weight $S U(3)$ irreps [27] of the Elliott and the proxy-SU(3) symmetry $[15,25]$. The plots appear in Figs. 1-6. The proton and neutron numbers indicated, identify the possible regions on the nuclear chart, where one may see coexisting structures, with drastically different shapes, lying close in energy.

Examples of shape coexistence appear in every of the above proton or neutron regions. For instance we see parity inversion in neutron numbers $N=7-8$ in the Be isotopes [37, 38], inversion of states at neutron numbers $N \geq 17$ in the $\mathrm{Mg}$ isotopes [3-5], a comeback of the HO magic numbers in the deformation parameter $\beta$ in Ni isotopes among neutron numbers $34 \leq N \leq 40$ [15], shape coexistence in the $\mathrm{Se}, \mathrm{Kr}, \mathrm{Sr}, \mathrm{Zr}$ isotopes at proton numbers $Z=34,36,38,40$ respectively [2], shape coexistence among $60 \leq N \leq 70$ in the $\mathrm{Sn}$ isotopes [39], or after $N=60$ in $\mathrm{Kr}$ isotopes [40], shape coexistence in the rare earths among proton numbers $60 \leq Z \leq 70$ [2], shape coexistence among $96 \leq N \leq 110$ in the $\mathrm{Hg}$ isotopes and fission isomers around $N=145$ in the superheavies [41].

Generally it is not implied that one should see shape coexistence in each and every isotope of the map in Fig. 25 of Ref. [15], but instead it is stated that the regions marked in this map are the widest possible islands of shape coexistence predicted by the dual-shell mechanism.

\section{Conclusions}

A unified view of the islands of inversion and of shape coexistence has been described. The mechanism supposes that the coexisting structures can occur when the proton or the neutron deformation due the valence spin-orbit like shell is larger than the one of the valence harmonic oscillator shell. This requirement is satisfied in certain islands on the nuclear chart, which coincide with the data about the islands of inversion and the islands of shape coexistence [2].

Nevertheless, although the dual-shell mechanism is promising, since it explains the manifestation of shape coexistence in various well-known paradigm nuclei, it is not at all a complete theory. Thus we do not expect to observe

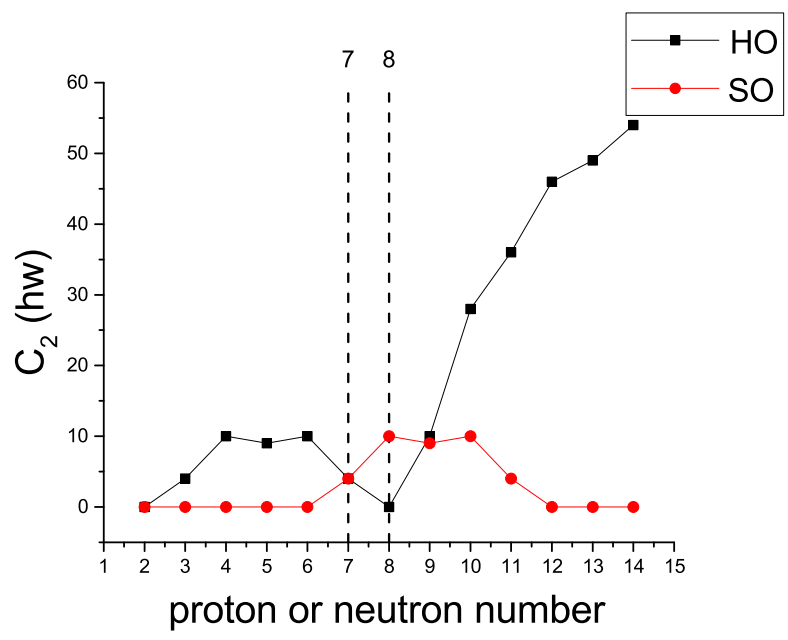

Figure 1. The eigenvalues of the second order Casimir operator of the $S U(3)$ algebra versus the proton $(Z)$ or neutron number $(N)$. Equation $(3)$ has been used along with the irreps $(\lambda, \mu)$ of Table 1 of Ref. [15]. Shape coexistence is predicted among proton or neutron numbers $7-8$.

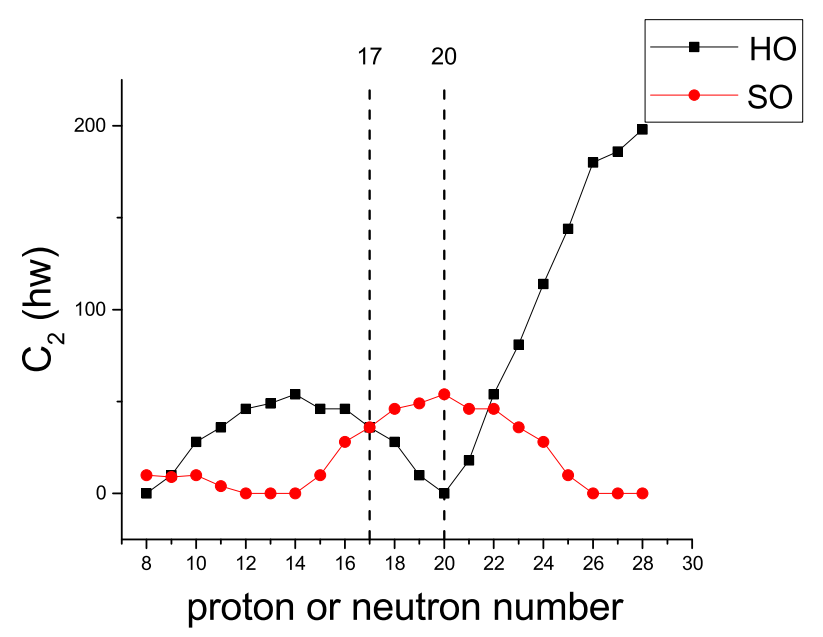

Figure 2. The same as Fig. 1. An island of shape coexistence is predicted within proton or neutron numbers $17-20$.

shape coexistence in each an every nucleus of the map of Fig. 25 of Ref. [15]. Much has to be done. The effect of the:

a) proton-neutron interaction,

b) merging of the spin-orbit like shell with the harmonic oscillator shell,

c) realistic interactions in the Hamiltonian (pairing and spin-orbit interaction for instance)

on the predicted islands of shape coexistence, has not been examined yet. The problem is that although studies for the these interactions within the Elliott $S U(3)$ framework began in the late 60's [23], they have never been completed. This dual-shell mechanism appears as a motivation to keep on studying the Elliott $S U(3)$ symmetry, because 


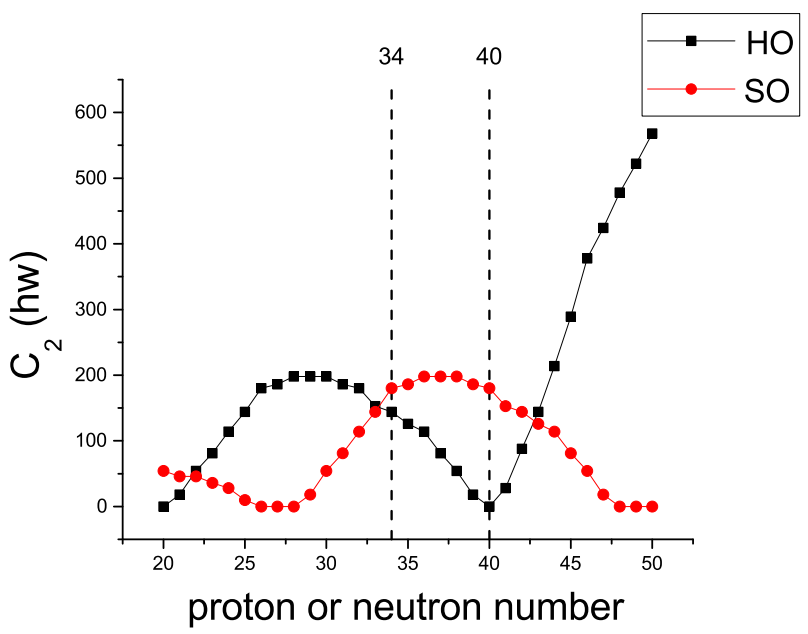

Figure 3. The same as Fig. 1. An island of shape coexistence is predicted within proton or neutron numbers $34-40$.

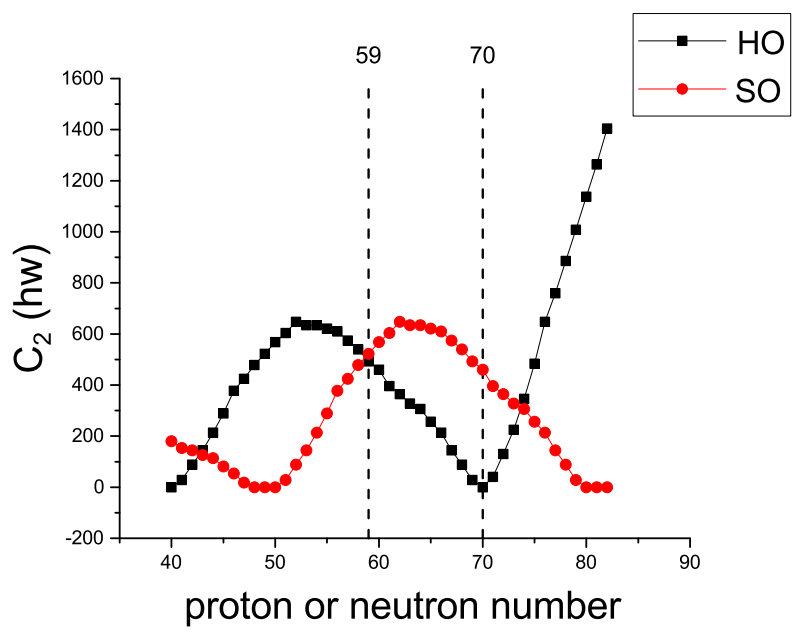

Figure 4. The same as Fig. 1. An island of shape coexistence is predicted within proton or neutron numbers $59-70$.

it is likely to give elegant solutions into burning problems of nuclear structure.

\section{Acknowledgements}

This research is co-financed by Greece and the European Union (European Social Fund- ESF) through the Operational Programme "Human Resources Development, Education and Lifelong Learning" in the context of the project "Reinforcement of Postdoctoral Researchers- $2^{\text {nd }}$ Cycle" (MIS-5033021), implemented by the State Scholarships Foundation (IKY).

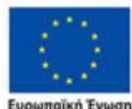

Operational Programme Human Resources Development Education and Lifelong Learning co-financed by Greece and the European Union

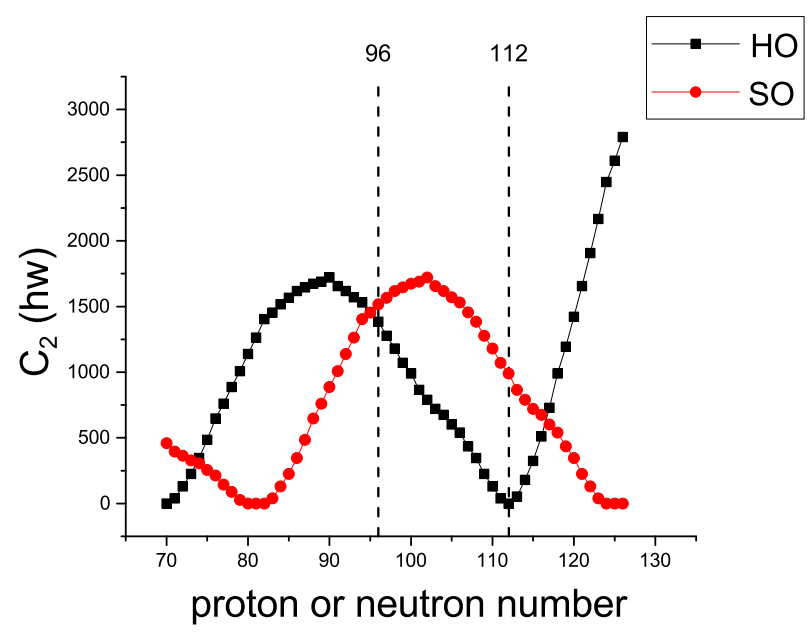

Figure 5. The same as Fig. 1. An island of shape coexistence is predicted within proton or neutron numbers $96-112$.

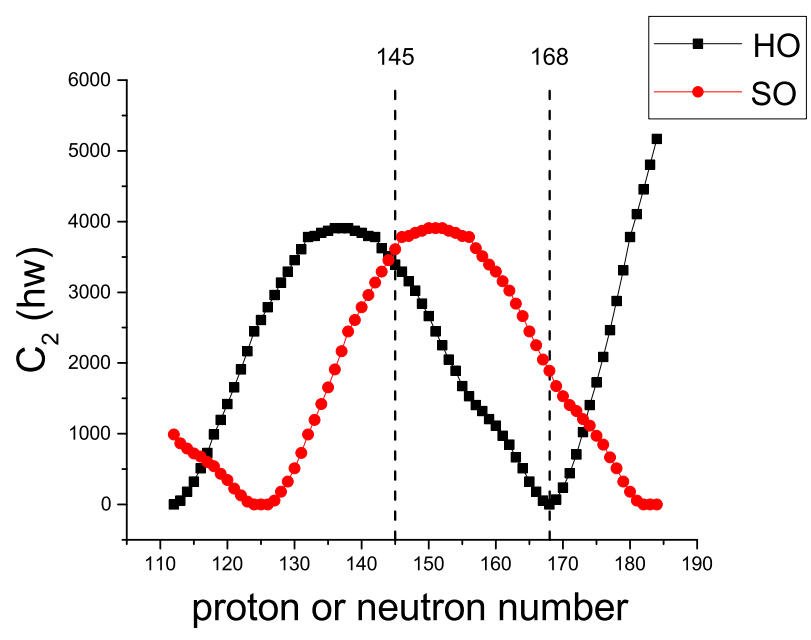

Figure 6. The same as Fig. 1. An island of shape coexistence is predicted within proton or neutron numbers $145-168$.

\section{References}

[1] H. Morinaga, Phys. Rev. 101, 254 (1956)

[2] K. Heyde, J.L. Wood, Rev. Mod. Phys. 83, 1467 (2011)

[3] P. Himpe, G. Neyens, D. Balabanski, G. Bélier, J. Daugas, F. de Oliveira Santos, M.D. Rydt, K. Flanagan, I. Matea, P. Morel et al., Phys. Lett. B 658, 203 (2008)

[4] M. Kowalska, D.T. Yordanov, K. Blaum, P. Himpe, P. Lievens, S. Mallion, R. Neugart, G. Neyens, N. Vermeulen, Phys. Rev. C 77, 034307 (2008)

[5] G. Neyens, M. Kowalska, D. Yordanov, K. Blaum, P. Himpe, P. Lievens, S. Mallion, R. Neugart, N. Vermeulen, Y. Utsuno et al., Phys. Rev. Lett. 94, 022501 (2005) 
[6] W. Schwerdtfeger, P.G. Thirolf, K. Wimmer, D. Habs, H. Mach, T.R. Rodriguez, V. Bildstein, J.L. Egido, L.M. Fraile, R. Gernhäuser et al., Phys. Rev. Lett. 103, 012501 (2009)

[7] K. Wimmer, T. Kröll, R. Krücken, V. Bildstein, R. Gernhäuser, B. Bastin, N. Bree, J. Diriken, P. Van Duppen, M. Huyse et al., Phys. Rev. Lett. 105, 252501 (2010)

[8] M.G. Mayer, Phys. Rev. 74, 235 (1948)

[9] O. Haxel, J.H.D. Jensen, H.E. Suess, Physical Review 75, 1766 (1949)

[10] A. Poves, Shape Coexistence and Islands of Inversion Monopole vs Multipole, in Proceedings of the Ito International Research Center Symposium "Perspectives of the Physics of Nuclear Structure" (Journal of the Physical Society of Japan, 2018)

[11] B.A. Brown, Physics 3, 104 (2010)

[12] F. Nowacki, A. Poves, J. Phys.: Conference Series 966, 012023 (2018)

[13] E. Caurier, F. Nowacki, A. Poves, Phys. Rev. C 90, 014302 (2014)

[14] O. Sorlin, M.G. Porquet, Progr. Part. Nucl. Phys. 61, 602 (2008)

[15] A. Martinou, D. Bonatsos, T.J. Mertzimekis, K.E. Karakatsanis, I.E. Assimakis, S.K. Peroulis, S. Sarantopoulou, N. Minkov, European Physical Journal A 57, 84 (2021)

[16] P. Federman, S. Pittel, Phys. Lett. B 69, 385 (1977)

[17] P. Federman, S. Pittel, Physics Letters B 77, 29 (1978)

[18] P. Federman, S. Pittel, Phys. Rev. C 20, 820 (1979)

[19] K. Heyde, P.V. Isacker, R. Casten, J. Wood, Phys. Lett. B 155, 303 (1985)

[20] J.P. Elliott, Proceedings of the Royal Society of London. Series A. Mathematical and Physical Sciences 245, 128 (1958)

[21] J.P. Elliott, Proceedings of the Royal Society of London. Series A. Mathematical and Physical Sciences 245, 562 (1958)

[22] J.P. Elliott, M. Harvey, Proceedings of the Royal Society of London. Series A. Mathematical and Physical Sciences 272, 557 (1963)

[23] J.P. Elliott, C.E. Wilsdon, Proceedings of the Royal Society of London. Series A. Mathematical and Physical Sciences 302, 509 (1968)

[24] D. Bonatsos, I.E. Assimakis, N. Minkov, A. Martinou, R.B. Cakirli, R.F. Casten, K. Blaum, Phys. Rev. C 95, 064325 (2017)
[25] D. Bonatsos, I.E. Assimakis, N. Minkov, A. Martinou, S. Sarantopoulou, R.B. Cakirli, R.F. Casten, K. Blaum, Phys. Rev. C 95, 064326 (2017)

[26] D. Bonatsos, European Physical Journal A 53, 148 (2017)

[27] A. Martinou, D. Bonatsos, N. Minkov, I.E. Assimakis, S.K. Peroulis, S. Sarantopoulou, J. Cseh, European Physical Journal A 56, 239 (2020)

[28] A. Martinou, D. Bonatsos, K.E. Karakatsanis, S. Sarantopoulou, I.E. Assimakis, S.K. Peroulis, N. Minkov, European Physical Journal A 57, 83 (2021)

[29] H.J. Lipkin, Lie groups for pedestrians (Dover, New York, 2002)

[30] J. Draayer, K.J. Weeks, Ann. Phys. 156, 41 (1984)

[31] J.P. Draayer, Algebraic approaches to nuclear structure: Interacting boson and fermion models (Harwood Academic Publishers, New York, United States, 1993), Vol. 6 of Contemporary Concepts in Physics, chap. 7, pp. 423-546, ISBN 3-7186-0538-4

[32] P.O. Lipas, Algebraic approaches to nuclear structure : interacting boson and fermion models (Harwood Academic Publishers, 1993), Vol. 6 of Contemporary Concepts in Physics, chap. 2, pp. 47-12

[33] A. Bohr, B.R. Mottelson, Nuclear Structure, Vol. II (World Scientific Publishing Company, 1998)

[34] O. Castaños, J.P. Draayer, Y. Leschber, Zeitschrift für Physik A Atomic Nuclei 329, 33 (1988)

[35] D.J. Rowe, G. Thiamova, J.L. Wood, Phys. Rev. Lett. 97, 202501 (2006)

[36] D. Bonatsos, A. Martinou, S. Sarantopoulou, I.E. Assimakis, S.K. Peroulis, N. Minkov, Eur. Phys. J. Spec. Top. 229, 2367 (2020)

[37] Y. Hirayama, T. Shimoda, H. Izumi, H. Yano, M. Yagi, A. Hatakeyama, C. Levy, K. Jackson, H. Miyatake, Nucl. Phys. A 746, 71 (2004)

[38] F. Cappuzzello, A. Cunsolo, S. Fortier, A. Foti, M. Khaled, H. Laurent, H. Lenske, J. Maison, A. Melita, C. Nociforo et al., Phys. Lett. B 516, 21 (2001)

[39] J.L. Wood, K. Heyde, W. Nazarewicz, M. Huyse, P.V. Duppen, Phys. Rep. 215, 101 (1992)

[40] F. Flavigny, P. Doornenbal, A. Obertelli, J.P. Delaroche, M. Girod, J. Libert, T. Rodriguez, G. Authelet, H. Baba, D. Calvet et al., Physical Review Letters 118 (2017)

[41] P. Thirolf, D. Habs, Progr. Part. Nucl. Phys. 49, 325 (2002) 